\title{
Perspective
}

PERSPECTIVE Actualité en histoire de l'art

$1 \mid 2020$

Japon

\section{Tōkyō Underground - Le topos des avant-gardes japonaises au tournant des années 1960}

Tōkyō Underground - The Topos of the Japanese Avant-Gardes in the Late 1960s Tōkyō Underground - Der Topos der japanischen Avantgarde in den 1960er Jahren

Tōkyō Underground - Il topos delle avanguardie giapponesi durante la svolta degli anni ' 60

Tōkyō Underground - El topos de los vanguardistas japoneses en el cambio de los años 1960

\section{Kei Osawa}

\section{OpenEdition}

Journals

Édition électronique

URL : http://journals.openedition.org/perspective/19048

DOI : 10.4000/perspective. 19048

ISSN : 2269-7721

\section{Éditeur}

Institut national d'histoire de l'art

\section{Édition imprimée}

Date de publication : 5 juin 2020

Pagination : 267-280

ISBN : 978-2-917902-89-9

ISSN : $1777-7852$

\section{Référence électronique}

Kei Osawa, "Tōkyō Underground - Le topos des avant-gardes japonaises au tournant des années 1960 », Perspective [En ligne], 1 | 2020, mis en ligne le 30 décembre 2020, consulté le 25 janvier 2021. URL : http://journals.openedition.org/perspective/19048 ; DOI : https://doi.org/10.4000/ perspective. 19048 


\title{
Tōkyō Underground - Le topos des avant-gardes japonaises au tournant des années 1960
}

\author{
Kei Osawa
}

À partir de la première moitié des années 1950, le monde de l'art japonais est traversé par une succession d'événements désormais bien connue. Alors déchiré, comme dans nombre d'autres pays, par une nouvelle crise de la représentation - opposant les tenants de l'abstraction, du réalisme socialiste et du surréalisme -, ses problématiques et ses polémiques sont court-circuitées par une nouvelle génération d'artistes qui, au moyen d'actes directs - performances, actions, happenings, events - redéfinissent le champ des pratiques artistiques et la notion même d'œuvre. L'histoire de ces pratiques, depuis les collectifs Jikken Kōbō 実験工房 (Atelier expérimental) et Gutai Bijutsu Kyōkai 具体美術 協会 (Association pour l'art concret) jusqu'à Zero Jigen ゼロ次元 (Dimension zéro), a été rapportée dans nombre d'études et d'expositions au Japon et ailleurs ${ }^{1}$. Ce faisant, ces performances (et leurs auteurs) ont été progressivement abstraites de leur contexte de production, avec pour conséquence une vision singulièrement réductrice de ces artistes et collectifs, présentés comme des personnalités uniques ou des mouvements radicaux dressés contre les masses. Mais ceux-ci évoluaient dans un milieu original, celui de subcultures (parfois de contre-cultures) inspirées par les générations contemporaines en Asie $^{2}$, en Europe ${ }^{3}$ et aux États-Unis ${ }^{4}$. Si le contexte sociopolitique de cette période a été analysé en détail, aucune étude n'a été consacrée à délimiter ce milieu particulier, généralement réduit à une atmosphère, à un air du temps, à une série de modes passagères. Une meilleure connaissance de la modalité d'implantation de ces subcultures dans le monde de l'art japonais enrichit notre compréhension de ce moment décisif dans l'histoire culturelle de l'après-guerre, ainsi que celle de leur apport dans la production artistique actuelle. Partant de cette hypothèse, le présent article vise à analyser en détail, autour de la notion d'underground, la création de ce topos artistique au milieu des années $1960 \mathrm{au}$ Japon, à partir d'un large corpus de textes et de publications éphémères. Il ne s'agit pas d'analyser les productions de tel ou tel artiste ou mouvement sous un prisme auteuriste, mais plutôt d'offrir une chronologie et une cartographie de l'underground tokyoüte ${ }^{5}$ qui mettent en évidence les réseaux artistiques et géographiques de l'époque, ainsi qu'une appréciation de ses apports artistiques, théoriques, sociétaux et politiques dans l'art contemporain japonais. 


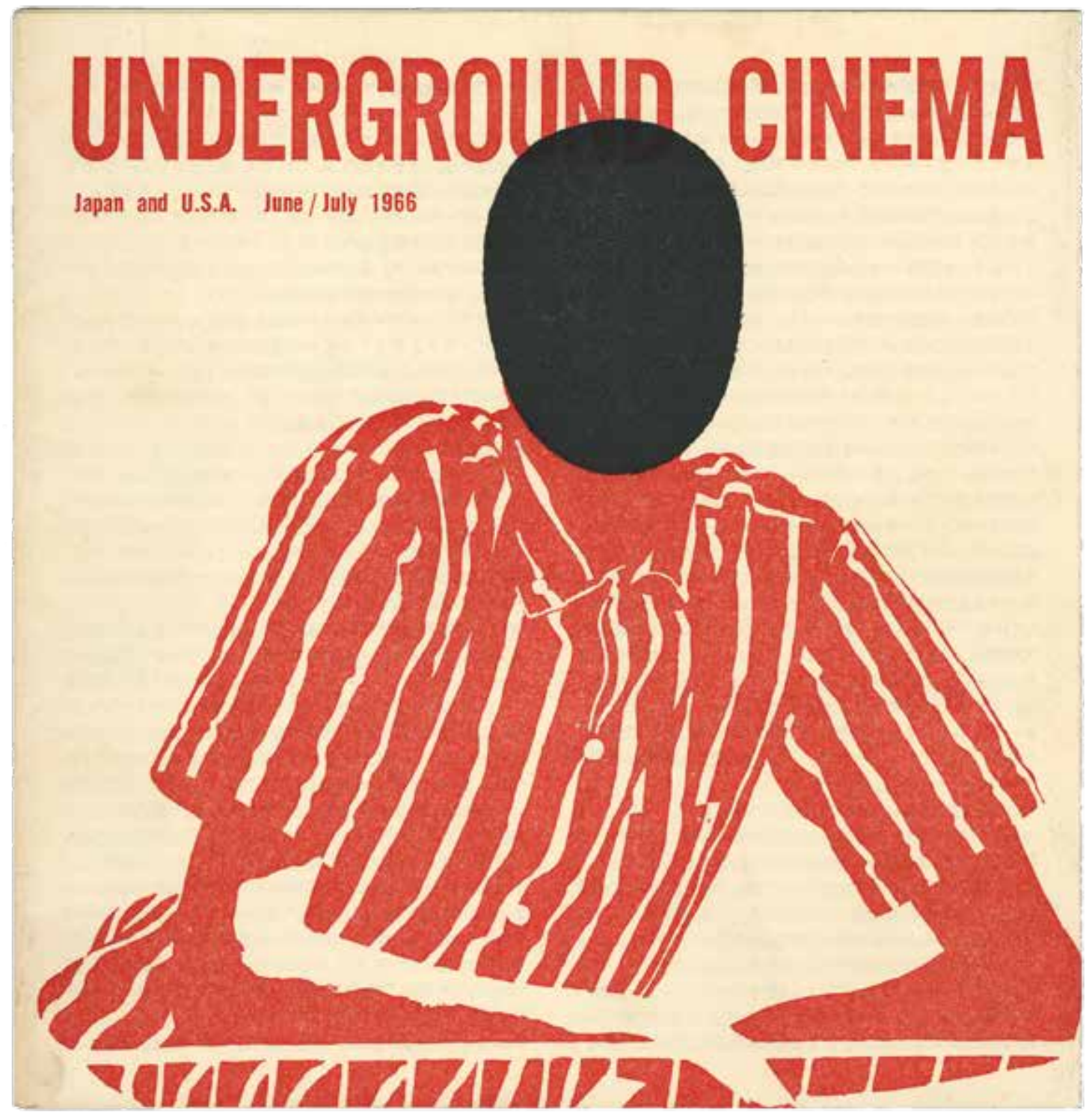

1a-b. Programme et billet d'Underground Cinema (29 juin - 2 juillet 1966) au Sōgetsu Kaikan Hall. Graphisme par Hosoya Gan.

\section{Avant-garde et underground}

Lorsqu'en 1968 l'artiste néodada Shinohara Ushio (né en 1932 à Tōkyō) publie précocement son autobiographie Zen'ei no michi [La voie de l'avantgarde $]^{6}$, le terme d'avant-garde (diversement rendu en japonais par la traduction littérale zen'e $i$ 前衛 ou la transcription avangyarudo アヴアンギャルド) est déjà investi de significations multiples remontant aux mouvements artistiques de l'ère Taishō (on employait alors, dans un sens voisin, le qualificatif shinkō 新興). Dès 1947, le Club des artistes d'avant-garde du Japon (Nihon avangyarudo bijutsuka kurabu 日本アヴアンギャルド美術家クラブ) s'était formé pour promouvoir la recherche sur la peinture d'avant-garde, et en 1948 un Groupe de recherche sur l'art d'avant-garde (Abangyarudo geijutsu kenkyūkai アバンギャルド芸術研究会) avait vu le jour, réunissant artistes, écrivains et critiques. Okamoto Tarō 岡本太郎 (1911-1996), 
figure de proue de l'art d'après-guerre, participait aux deux cercles. C'est Okamoto qui écrit la quatrième de couverture du livre de Shinohara, adoubant ainsi le représentant d'une génération nouvelle se situant à la pointe des expérimentations artistiques.

Or le terme est déjà éculé. L'examen détaillé des principaux journaux japonais révèle qu'en

䒹月シ*จテーク

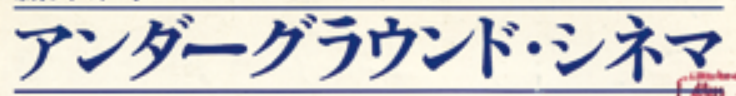
UNDERGROUND CINEMA 6ค 29日-30日 7ค1日-2日 เง 6.30 p.m (28ts $2.30,6.30 \mathrm{pm})$ 고슨 $-\pi$

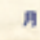
1968, le terme underground et ses variantes (notamment la transcription andäguraundo アンダーグラウンド et son abréviation angura アングラ) sont omniprésents dans les médias et la critique, rivalisant avec avangyarudo au point de l'écarter. Le lexique annuel des termes contemporains fournit dans son édition de 1969 une notice pour angura: « Mot de provenance anglaise forgé au Japon, abréviation du terme underground. Adjectif qualifiant les arts créés et présentés en souterrain, dans des lieux non éclairés par le soleil, non publics. L'angura provient du cinéma, mais il s'applique au théâtre, aux arts, à la danse, et a finalement donné les angura zoku アングラ族 ( "tribus underground ${ }^{7} »$ ). Au moment de son apparition, il s'opposait au commercialisme, tentait des expérimentations nouvelles, et impliquait parfois l'engagement de sympathisants dans des activités souterraines pour des raisons politiques, mais il recouvre aujourd'hui de nombreux éléments non identifiés ${ }^{8}$. " Les lexicographes saisissent déjà, non sans ironie, le caractère flou des modes d'expression et des styles de vie rassemblés sous cet emprunt linguistique à l'anglais. Mais le terme désigne une réalité sociale tangible : inspirés par le cinéma expérimental, des artistes s'engagent dans des modes de production indépendants pour créer des œuvres exigeantes et non commerciales, parfois contestataires, qui engendrent à leur tour des mœurs originales. Certaines définitions précisent que ces modes d'expression sont d'avant-garde : ce qualificatif historique se trouve ainsi absorbé par un terme spatial à l'extension plus large et au sens plus flou.

Dans l'édition de 1968, le même lexique relevait déjà un terme étroitement associé à l'underground: füten フーテン`. Désignant initialement sur un registre familier les pathologies mentales ou un accès de délire ${ }^{10}$, ce mot est détourné pour qualifier les hippies japonais et ceux qui s'y reconnaissent, en insistant sur leur mode de vie nocturne, leurs pratiques déviantes et leur oisiveté. Il connaîtra une popularité durable grâce à la série de films Otoko wa tsurai yo 男はつらいよ [C'est dur d'être un homme] réalisés par Yamada Yōji 山田洋次 (né en 1931) à partir de 1969, dans lesquels le protagoniste se voit affublé de ce qualificatif.

À la fin des années 1960, les lexiques spécialisés dans l'argot angura ne manquent pas ${ }^{11}$ : une fois l'underground établi parmi les jeunes générations, il s'agit d'asseoir une langue nouvelle, fondée sur des emprunts à l'anglais, des détournements et des contractions de termes japonais, ainsi que des références à un vocabulaire politique d'extrême gauche.

\section{Le vecteur du cinéma}

On retient généralement de l'angura cette atmosphère vague et sulfureuse qui flotte dans les quartiers jeunes de Tōkyō à la fin des années 1960, perceptible dans la photographie et le cinéma de l'époque. Or son origine peut être précisément retracée. Le terme est employé pour la première fois de manière notable par le réalisateur, photographe et critique Kanesaka Kenji 金坂健二 (1934-1999) lorsqu'il organise, du 29 juin au 2 juillet 1966 au Sōgetsu Kaikan 草月会館 ${ }^{12}$, sous le titre Underground Cinema, une série de projections de films expérimentaux en 8 et 16mm rapportés de son séjour aux États-Unis (fig. la-b). 


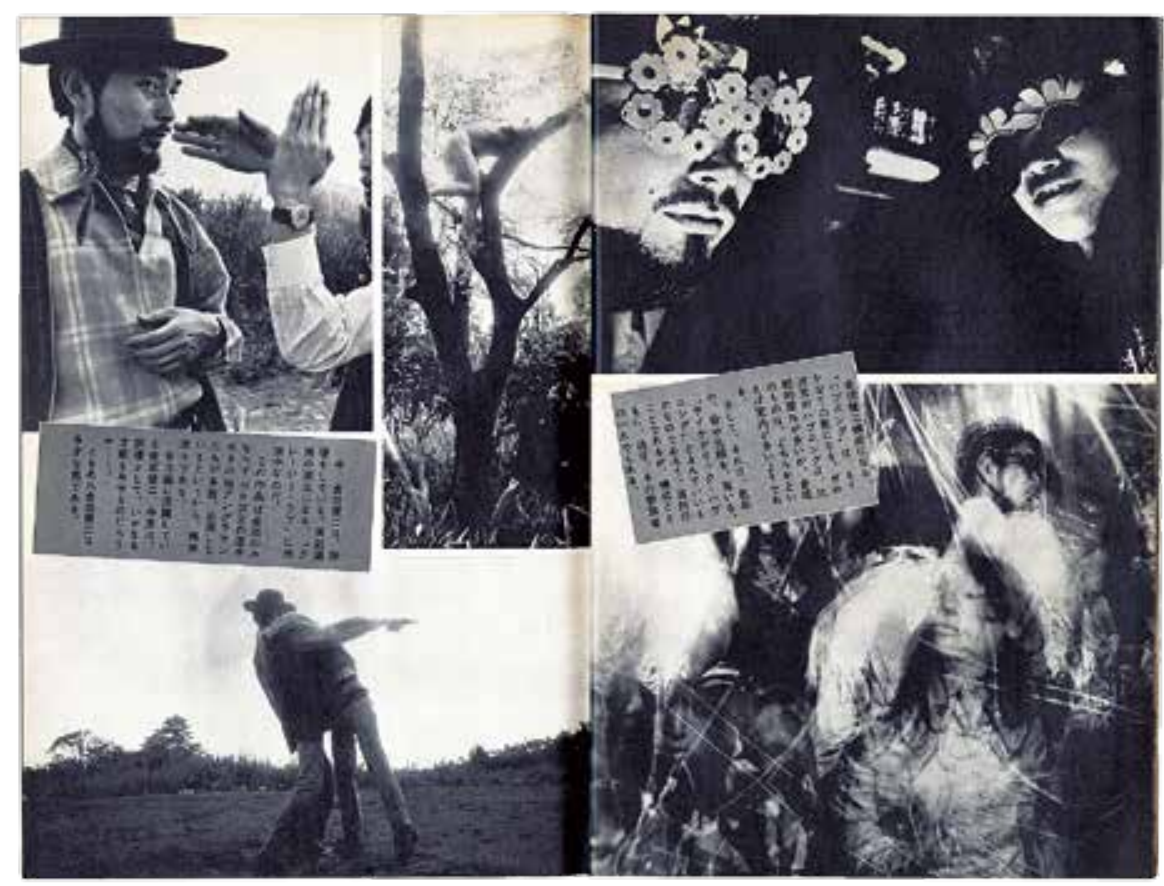

2a-b. «Kanesaka Kenji, Champion de l'underground", article publié dans le numéro spécial « Angura '68 shokku-hen »アングラ'68 ショック篇 [Underground 68, volume choc] de la revue Bessatsu Kinema Junpō (août 1968), avec un portrait de Takakura Ken en couverture, retouché et mis en page par Yokoo Tadanori.
Sont projetés des films d'Iimura Takahiko 飯村隆彦 (né en 1937), Stan Brakhage, Donald Richie, Joe Sedelmaier, Carl Linder, Robert Nelson et Kanesaka lui-même. Sur le programme figurent des textes de Jonas Mekas, de Robert Brown et du critique Tōno Yoshiaki 東野芳明 (1930-2005), exclusivement sur le cinéma. Kanesaka réitère sa présentation l'année suivante, du 8 au 14 mars, toujours au Sōgetsu Kaikan. L'Underground Film Festival présente alors les films de Jonas Mekas, Stan Vanderbeek, Bruce Baillie, Jud Yalkut, Kanesaka et Ōbayashi Nobuhiko 大林宣彦 (1938-2020). Les textes imprimés dans le programme (mis en page par Hosoya Gan 細谷嚴 [né en 1935], comme pour l'édition précédente), signés par Stan Vanderbeek, Iimura Takahiko, Kanesaka Kenji et le réalisateur Hani Susumu 羽仁進 (né en 1928), traitent notamment de l'expanded cinema et de l'intermedia, toujours selon l'axe nippo-américain, mais en dépassant le paradigme strictement cinématographique pour s'ouvrir aux autres disciplines artistiques ${ }^{13}$.

La diffusion du cinéma underground, sous influence américaine et par le biais de Kanesaka, est alors fulgurante. La revue spécialisée Eiga hyōron 映画評論 [Critique de cinéma] sous la houlette de l'éditeur et critique Satō Shigechika 佐藤重臣 (1932-1988) traite le sujet dans ses numéros de juillet et de septembre 1966, et le livre de Sheldon Renan, An Introduction to the American Underground Film, est traduit en japonais en $1969^{14}$. Durant l'été 1968, la revue de cinéma Kinema Junpōキネマ旬報 [The Movie Times] publie en juin ${ }^{15}$ puis en août ${ }^{16}$ deux numéros spéciaux intégralement consacrés à l'angura. La situation a changé : le cinéma n'est qu'un secteur parmi d'autres dans le vaste souterrain agitant la capitale. Ainsi le numéro de juin présente-t-il pêle-mêle les « chefs-d'œuvre de l'affiche psychédélique [de théâtre et de cinéma] ", les troupes de théâtre underground, les " huit samouraïs de l'underground ${ }^{17}$ ", des photographies du théâtre et du cinéma 


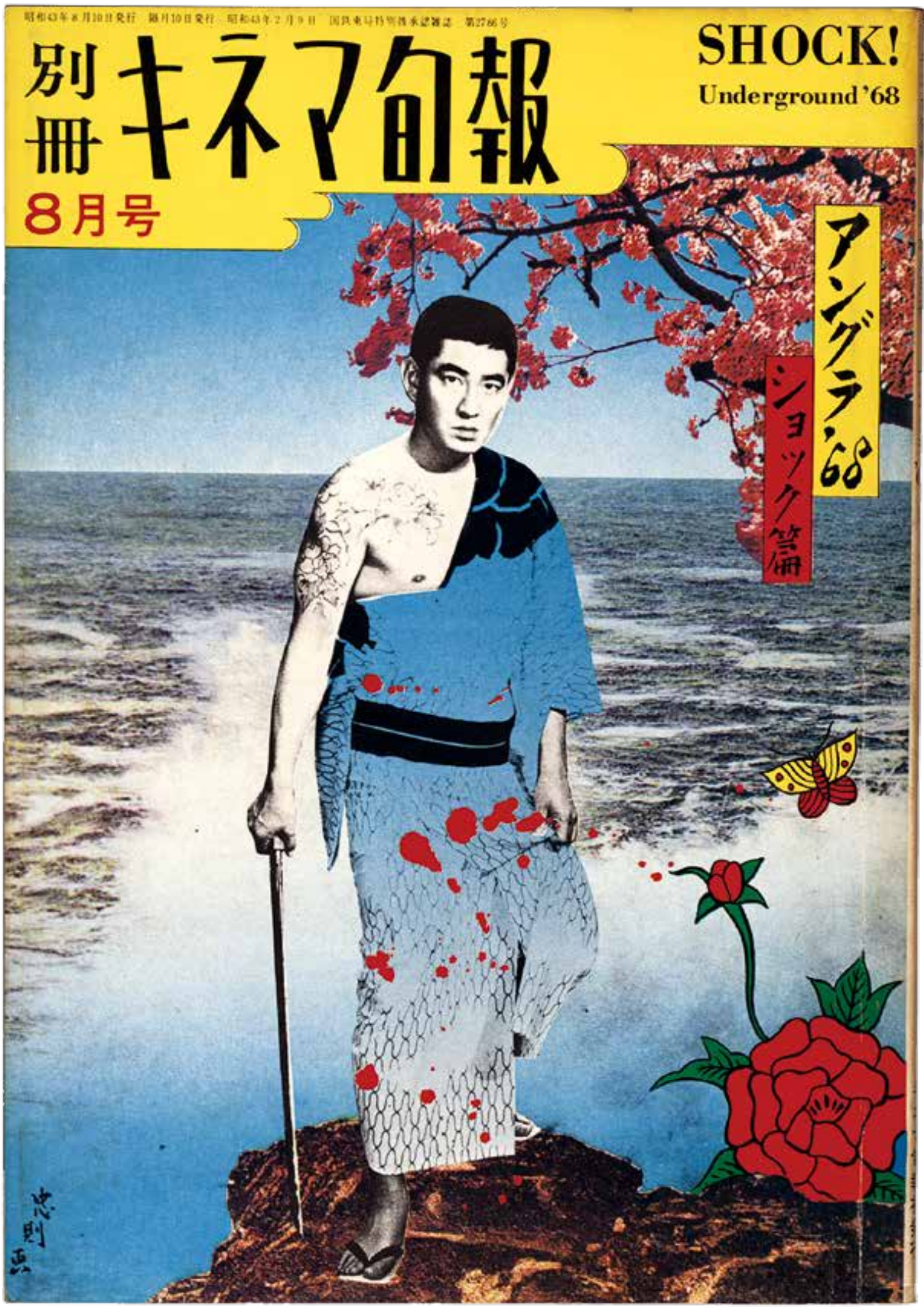


underground, des manga, une table ronde sur les arts underground, un guide des lieux underground, et un article spécial sur le psychédélique et les drogues. Le numéro d'août poursuit cette ouverture vers les autres disciplines en accentuant le caractère érotique et contestataire de nombre d'œuvres présentées. Apparaissent ainsi le body painting, les happenings de Zero Jigen, un dialogue entre Yokoo Tadanori et l'acteur Takakura Ken 高倉 健 (1931-2014), des articles sur la nudité, l'homosexualité, les drogues en lien avec l'underground, puis une section consacrée au cinéma underground, comme pour donner une cohérence, en fin de volume, à un numéro par ailleurs disparate (fig. 2a-b).

\section{" Les explorateurs des zones intermédiaires"}

Cette dispersion reflète la prolifération rapide de l'underground dans le paysage tokyoïte. L'artiste et musicien Tone Yasunao 刀根康尚 (né en 1935) est le premier à dresser un tableau systématique des arts underground au Japon pour en mesurer la portée réelle, loin des stéréotypes sulfureux qui s'y rapportent et que les principaux représentants du mouvement réfutent. La revue Bijutsu techō 美術手帳 [Cahier d'art] offre dans son numéro de novembre 1967 un dossier sur les arts underground au Japon (l'autre dossier portant sur Pierre Bonnard), dans lequel Tone publie son " Underground Arts Diagram ${ }^{18}$ " (fig. 3). Cet arbre généalogique mériterait une étude à part entière : la colonne de gauche regroupe les principaux représentants américains de l'underground, tandis que les autres cases, en bas de l'arbre, représentent les diverses mouvances japonaises avec leurs ancêtres (qui pour certains remontent jusqu'au wagnérisme, au vaudeville, au zen et même au vajrayāna) et leurs interrelations. Si nous ne pouvons nous attarder ici sur tous les groupes figurant sur le diagramme, relevons les disciplines artistiques représentées dans la partie japonaise : la performance, le happening, l'event, l'action, le théâtre, l'intermedia, le butō, la musique, le cinéma, l'édition de revues.

L'absence de la littérature est patente, non seulement dans ce dossier mais dans la plupart des études se rapportant à l'underground, alors même que des classiques de l'après-guerre tels que Sho o suteyo machi e deyō 書を捨てよ町へ出よう [Jetez vos livres, sortez dans la rue] (1967) de Terayama Shūji étaient déjà publiés. Si c'est le légendaire roman Kachikujin yapū 家畜人ヤプー [Yapou, bétail humain] ${ }^{19}$, publié en 1956, qui acquiert le statut de chef-d'œuvre underground, on relève alors plutôt des poètes désormais inconnus, telle Hoshino Mari 星野マリ qui publie en 1969 Shinjuku chikadō no shi 新宿地下道の詩 [Poèmes des voies souterraines de Shinjuku]. Ceci s'explique par le scepticisme fondamental de l'underground vis-à-vis du langage, compris - sous l'influence d'une lecture biaisée de McLuhan - comme un système de domination. Tone cite dans son article un entretien avec Kanesaka : « Le domaine dont les artistes pourront maintenant traiter, auquel ils pourront faire face, se rapporter, je pense que c'est la perception, ce n'est pas la conscience... Et puis le langage est hiérarchique, la littérature a un aspect inébranlable. Je pense que tout ça relève du système. C'est par rapport à ce système que nous situons dans l'underground un anti-système ${ }^{20}$. ". De fait, si l'on écarte la bande dessinée ${ }^{21}$, la littérature n'est mobilisée que sous son versant performatif, intégrée à des dispositifs scéniques.

La parole a ainsi une maigre présence dans la musique underground de l'époque, notamment dans le jazz et les musiques improvisées ${ }^{22}$, mais elle prendra toute sa place dans un genre inattendu mais fondamental pour sa dimension politique : le folk japonais ${ }^{23}$. Nous y reviendrons. C'est le théâtre qui se fait le porte-étendard de l'underground, les principales troupes indépendantes (notamment Tenjō Sajiki 天井栈敷, mené par Terayama Shūji et Jōkyō Gekijō 状況劇場, mené par Kara Jūrō, mais on en compte une 

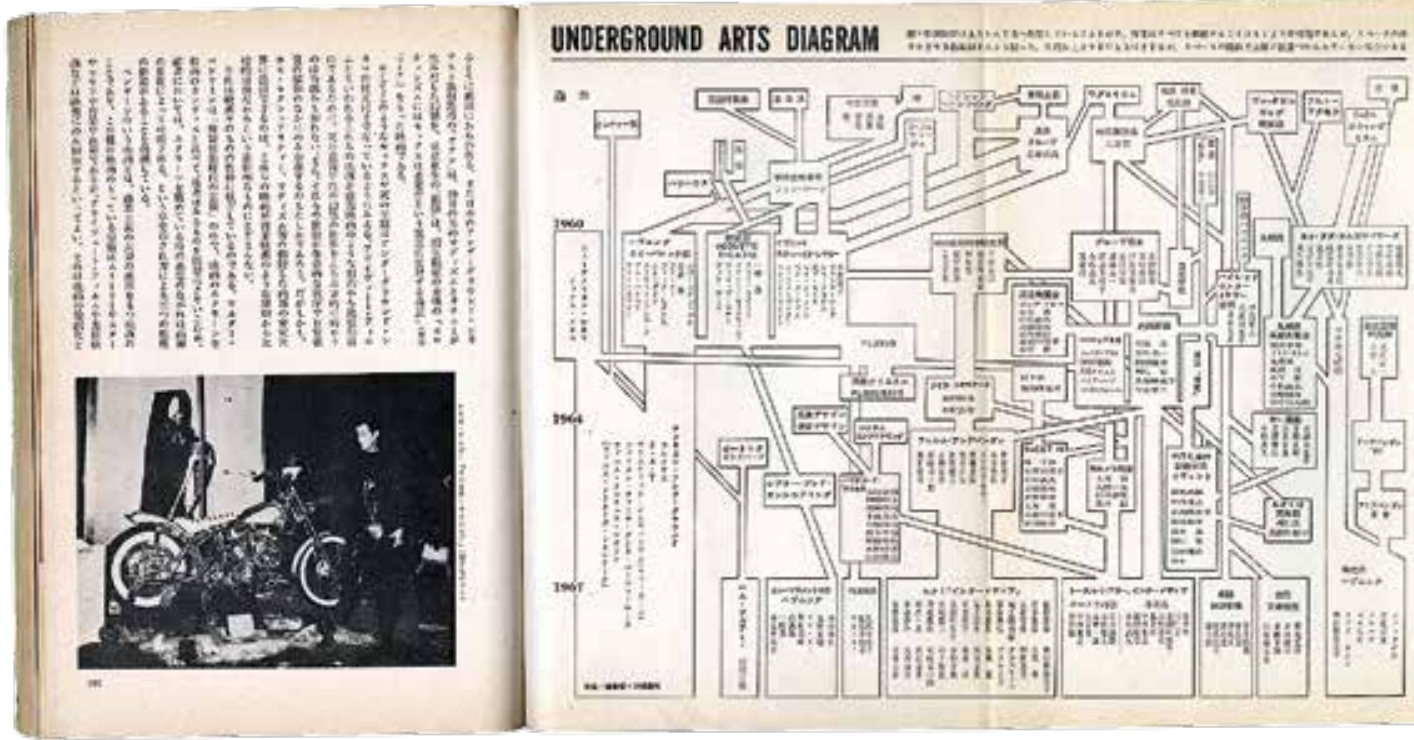

dizaine d'autres) quittant rapidement les théâtres pour investir des parcs, des temples et des sanctuaires puis éventuellement les rues ${ }^{24}$. C'est dans ce contexte que le théâtre en vient à incarner ce que Nishidō Kōjin 西 3. Tone Yasunao, "Underground Arts Diagram », publié dans Bijutsu techō en novembre 1967. Sur la page de gauche, une image tirée de Scorpio Rising (1964) de Kenneth Anger.

堂行人 (né en 1955) nomme le " paradigme underground ${ }^{25}$ ". Il exerce également un rôle majeur dans la circulation de l'information et d'un style graphique propre, par le biais de ses publications éphémères (affiches, bulletins, tracts, programmes) ${ }^{26}$. Dans son étude, Tone situe l'apport du butō de Hijikata Tatsumi 土方异 (1928-1986) dans son ambition d'un théâtre synthétique : il intègre à sa danse les productions artistiques de ses contemporains pour produire une œuvre totale, incarnation possible de l'intermedia. Car dans sa perspective d'intégrer la perception dans de nouvelles formes indépendantes d'expression à même de fédérer une communauté internationale, l'underground est indissociable de l'intermedia : Tone affirme que c'est par des productions artistiques totales que l'underground peut produire non pas une nouvelle culture, mais une culture autre.

Naturellement, le cinéma conserve sa prééminence et s'assure un ancrage solide dans l'underground: on organise un Film art festival, résultant d'un concours, au Sōgetsu entre 1967 et 1969 ; le Japan Filmmakers' Cooperative mené par Satō Shigechika est actif dès 1968, avant que Satō lui-même ne le quitte pour former en 1969 le Nihon andāguraundo sentā 日本アンダーグラウンド•センター [Centre underground japonais], duquel émanera en 1971 l’Underground Center sous la direction de Kawanaka Nobuhiro かわなかのぶひろ (né en 1941).

Il est révélateur que ce mouvement culturel n'ait laissé que peu de traces apparentes dans le monde de l'architecture et le design. Une des rares références directes à l'underground dans le domaine de l'architecture consiste dans une publication pour le moins énigmatique de Takaguchi Yasuyuki 高口恭行 et Manabe Tsunehiro 真鍋恒博 intitulée Le troisième architecte $^{27}$. Les rares études sur la possibilité d'une transformation de ce que Kanesaka nomme le "système" sont sceptiques ${ }^{28}$. Dix ans plus tard, en 1982, lorsque la revue Kenchiku bunka 建築文化 [Culture architecturale] publie le numéro spécial «Plan Tōkyō underground ${ }^{29}$ " édité par un panel de spécialistes d'urbanisme et d'architecture, 

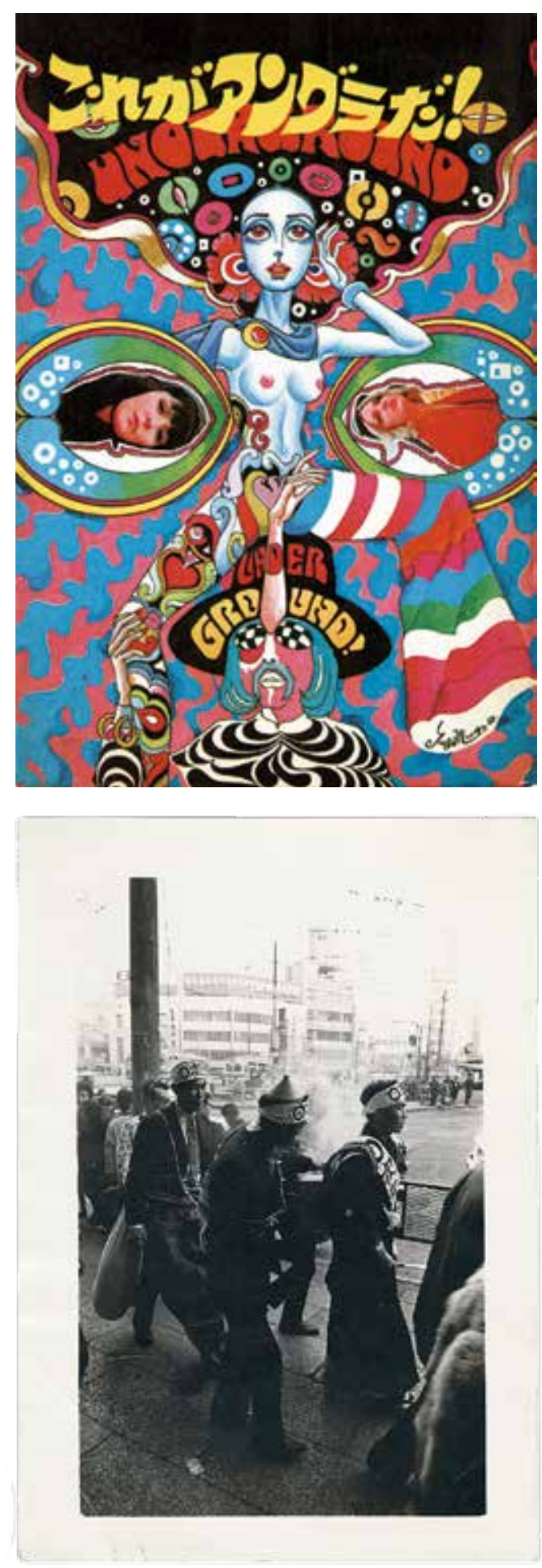

4a-c. Première de couverture de Korega angura da! これがアングラだ! [Voici l'underground !] et tirages originaux des photographies des membres du groupe Zero jigen pratiquant leurs rites, publiés en juillet 1968. malgré la reprise d'un vocabulaire fortement connoté ( "Tōkyō underground » angura Tōkyō アングラ東京, " underground mandala " angura mandala アングラ 曼茶羅, etc.), underground est entendu exclusivement comme un réseau d'infrastructures souterraines à rénover radicalement afin de soutenir la croissance soutenue de la mégalopole. Les lieux de l'underground sont uniquement traités comme des problèmes à résoudre : les places à plusieurs étages (comme celle qui se situe à la sortie ouest de la gare de Shinjuku) doivent être redessinées pour offrir un environnement plaisant pour le passage et le repos ${ }^{30}$, tandis que les passages souterrains qui forment une ville à part entière sous terre doivent être reconsidérés dans leur ensemble pour offrir " sécurité et santé31". Ceci porterait à appuyer la thèse des détracteurs de l'underground, selon lesquels celui-ci n'aurait été qu'une mode passagère ${ }^{32}$.

\section{Les lieux de l'underground}

Pourtant, l'underground était durablement inscrit dans des lieux physiques dans le quartier de Shinjuku, et de nombreux témoignages l'ont rapporté en temps réel $^{33}$. Un trio de journalistes publie en 1968 un guide sur les rituels de l'underground, ses représentants et ses lieux ${ }^{34}$ (fig. 4a-c). La même année, l'universitaire Fukasaku Mitsusada 深作光貞 (1925-1991), s'inspirant de la tradition japonaise de la " modernologie " (kōgengaku 考現学), publie une étude complète sur Shinjuku ${ }^{35}$ qui précise les modalités de l'installation de l'underground dans ce quartier.

Établi en 1698 à l'ouest d'Edo au sein du domaine de Naitō Wakasanokami 内藤若狭守, seigneur du fief de Takatō 高遠 dans la province de Shinano 信濃, comme relais de la Kōshū kaidō 甲州街道 (la route reliant Edo à la province de Kai), le poste de Naitō Shinjuku 内藤新宿 connaît une croissance mouvementée mais rapide. Dès le début, les propriétaires des commerces et des maisons closes d'Asakusa investissent massivement dans le développement du site, et le poste se remplit de relais, de restaurants, d'auberges et de maisons closes. À travers ses agrandissements successifs marqués par la construction de la gare ferroviaire en 1885, son accès après la guerre au statut de "sub-centre " (fukutoshin 副都心) de la capitale, puis le développement de la zone ouest en quartier de bureaux à partir de 1960, Shinjuku a gardé son caractère de lieu de transit : on va jusqu'à 


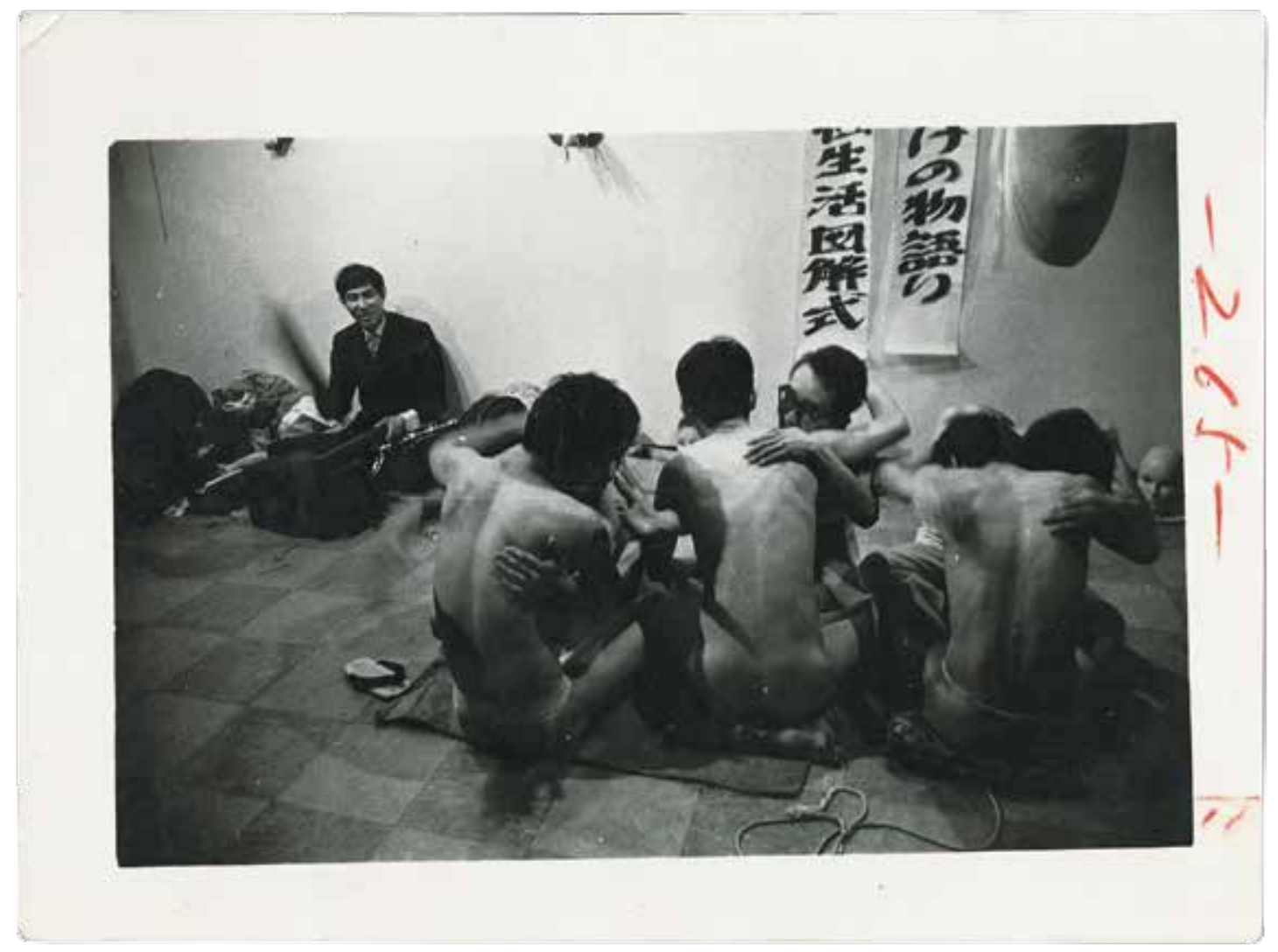

Asakusa, à Ginza les employés restent le soir se divertir dans des lieux huppés, mais on ne fait que passer à Shinjuku. La gare est un terminus avec de nombreux cafés et lieux de divertissements ouverts la nuit, et il s'y développe une industrie du sexe. Dès l'avant-guerre, c'est un quartier de théâtres. C'est un lieu de rassemblement des jeunes, bon marché, à proximité des universités Waseda et Musashino Bijutsu. L'absence de traditions locales comme à Ginza ou à Asakusa y a attiré une population hétéroclite, faite d'étudiants, d'artistes, d'activistes d'extrême gauche et de füten. Sur ces énergumènes, la presse est intarissable ${ }^{36}$.

Se développe rapidement une catégorie de publications spécialisées : les cartes de l'underground à Shinjuku repérant tous les lieux concernés (fig. 5a-b), une sorte de prolongement ludique à la cartographie imaginaire de Shinjuku que Terayama Shūji et le photographe Moriyama Daidō 森山大道 (né en 1938) offraient en 1966 en supplément du roman Aa kōya あ 〉、荒野 [Aa, terre vaine]. Son cœur, l'Art Theatre Shinjuku アート シアター新宿文化 (Āto shiatā Shinjuku bunka) ${ }^{37}$, fondé en 1962, se double le 20 juin 1967 d'une annexe souterraine, justement nommée Underground Scorpio アンダーグラウンド 蠍座 (Andāguraundo sasoriza). Cet établissement est le temple du cinéma indépendant, et noue à travers ses représentations théâtrales et de danse des liens avec les grands noms de la littérature, de la musique, de la danse, des arts et du graphisme. En 1967 ouvre le théâtre Modern Art モダンアート (Modan āto), baptisé « cave des adeptes de la sensation ", et qui offre cinq types de représentations : des nude shows, du théâtre d'avant-garde et de divertissement, des projections de films $8 \mathrm{~mm}$, des performances du moment. Même s'il produit de célèbres artistes de la performance, c'est le strip-tease 


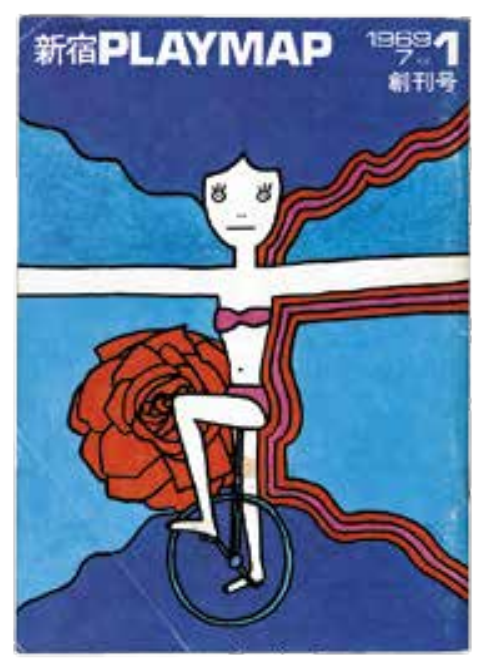

5a-b. Carte de Shinjuku publiée dans le premier numéro de Shinjuku Playmap (juillet 1969).

(nommé, non sans pédanterie, angura nude) qui attire le plus de monde, au grand dam de certains artistes qui y voient un dévoiement de l'underground à des fins commerciales. Autour de ces deux sites ouvrent une dizaine de cafés qui sont le repaire des füten, le plus connu étant Fūgetsudō 風月堂 qui rouvre en 1955 et où le vétéran Akiyama Kuniharu 秋山邦晴 (1929-1996) donne des concerts de disques de musique contemporaine. À l'extérieur, l'enceinte du sanctuaire Hanazono 花園神社 accueille la tente rouge de la troupe Jōkyō Gekijō.

Shinjuku est scindé en deux par la voie ferrée, et tous les lieux mentionnés se situent dans le quartier est. Les travaux menés pour faire de la zone ouest un nouveau quartier d'immeubles de bureaux aboutissent à la création d'une grande place étagée à la sortie ouest de la gare ${ }^{38}$. Initialement, cette place fait l'objet d'un véritable projet urbanistique ${ }^{39}$. Or son occupation pérenne par les füten qui s'y rassemblent pour chanter du folk pacifiste, ainsi que les violentes manifestations qui s'y déroulent, vont amener les autorités à modifier les modalités de son usage, ce qui, à terme, a pour conséquence d'éradiquer de l'espace public de Shinjuku toute trace visible de l'underground. Le 21 octobre 1968 une première manifestation est réprimée. Le 21 octobre 1969, une manifestation contre la guerre ${ }^{40}$ est à son tour violemment réprimée, à tel point que le quotidien Asahi titre dans son édition du lendemain : "Guérilla au cœur de Shinjuku ". De vifs débats sur la possibilité de voir, dans ce débordement, l'opportunité de créer un véritable forum divisent les intellectuels ${ }^{41}$, mais ils sont pris de court par les autorités. Les journaux rapportent alors que la police a rebaptisé la place de la sortie ouest de Shinjuku "passage ", et y a interdit tout rassemblement. Ce changement de dénomination signe la destruction symbolique de l'underground comme lieu public : celui-ci était toléré tant qu'il se cantonnait à des espaces privés du quartier est, mais son débordement sur la place publique du quartier ouest a signé son arrêt de mort. Cette transformation hâtive est symptomatique d'un certain état de la société japonaise dans son ensemble. En 1970, dans un article dressant le bilan de l'Exposition universelle du point de vue de l'architecture et du design, l'historien de l'architecture Fukuda Seiken 福田晴虔 observe qu'alors que la place de la sortie ouest de Shinjuku fut soudainement rebaptisée passage, l'opération sémantique inverse a été appliquée lors de l'Exposition universelle : on a rassemblé dans un espace baptisé " grande place " une masse d'individus sans solidarité préalable pour les faire interagir et les faire participer aux festivités préparées par les organisateurs. Or une telle production d' « harmonie " instantanée ne peut se faire sans un dispositif de pouvoir ${ }^{42}$.

Quoi qu'il en soit, le constat d'échec est implacable : dès l'été 1969 les médias rapportent que les füten désertent progressivement la place publique, et après décembre 1969 le mot n'apparaît même plus dans les journaux ${ }^{43}$.

\section{L'underground comme topos artistique}

Une fois l'underground physiquement aboli, demeurent ses représentations artistiques. Outre la photographie ${ }^{44}$, c'est au cinéma d'intégrer dans son monde fictionnel, cette fois-ci sous le mode de la représentation, l'underground qu'il a contribué à propager au Japon. L'année 1969 voit la sortie de Shinjuku dorobō nikki 新宿泥棒日記 [Journal d'un voleur 
de Shinjuku] par Ōshima Nagisa 大島渚 (1932-2013) qui a pour décor principal la librairie Kinokuniya, et de Bara no sōretsu 蔷薇の葬列 [Les funérailles des roses] par Matsumoto Toshio 松本俊夫 (1932-2017), dont le décor est campé dans le quartier gay de Shinjuku ${ }^{45}$. L'année suivante sort Shinjuku maddo 新宿マッド [Le fou de Shinjuku] par Wakamatsu Kōji 若松孝二 (1936-2012), sur un scénario d'Adachi Masao 足立正生. Ces films offrent un portrait de la génération underground rêvée par Kanesaka Kenji ${ }^{46}$.

Or l'underground en tant que topos structuré par des réseaux personnels et incarné par des styles et des modes plus ou moins stéréotypés ne survit pas à la disparition de ses lieux d'ancrage, comme l'atteste le clivage entre les artistes et les intellectuels qui participent à l'Exposition universelle d'Ōsaka en 1970 et ceux qui s'y opposent. Si les raisons de cette polémique sont complexes et les positions de chacun variées, il est clair que la participation de certains artistes underground à l'Exposition contribue à incorporer leur mode d'expression au mainstream. Ce dévoiement n'est pas uniquement politique (au sens du passage d'un mode de production indépendant vers une soumission aux entreprises et à l'État) : il est également artistique. Tone avait perçu le lien intrinsèque unissant underground et intermedia ; or l'Exposition a précisément appliqué le principe de l'intermedia à ses grandes productions multimédia, s'en appropriant les moyens à des fins tout autres. Cette rupture amorce la dissémination, à partir des années 1970, de l'underground vers d'autres quartiers de Tōkyō, souvent avec d'autres appuis institutionnels (notamment les grands magasins) qui transmettent en l'édulcorant cette subculture à un public plus large ${ }^{47}$.

Dès lors, quels sont les apports de l'angura aujourd'hui ? Son principal apport est structurel : il a initié une révolution dans la compréhension des mouvements artistiques japonais d'après-guerre en opérant un passage depuis une compréhension historiciste des avant-gardes vers une conception spatialisée de mondes distincts qui coexistent, au Japon et à l'étranger, œuvrant ensemble à des formes radicales d'expression. Dans un entretien accordé à Tone Yasunao, Katō Yoshihiro 加藤好弘 (1936-2018) de Zero Jigen affirme : «Zero jigen, ce n'est pas l'avant-garde. La question de savoir si c'est nouveau

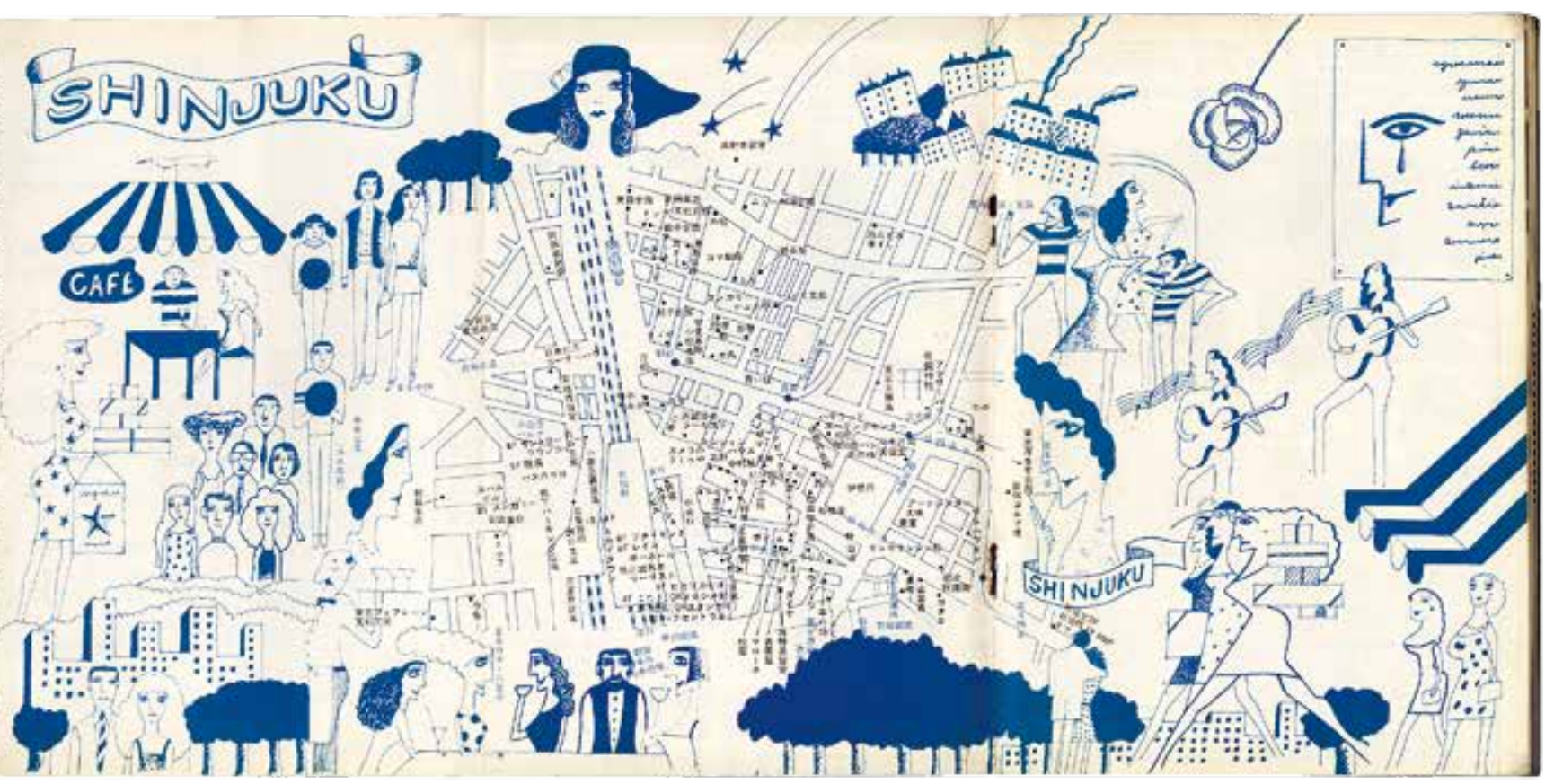


ou ancien ne m'intéresse pas vraiment... Bien sûr, comme j'habite le vingtième siècle, je suis perché à la pointe du vingtième siècle... Mais par exemple, si on prend pour axe des ordonnées l'histoire, c'est - vu depuis la pointe de cet axe - l'axe des abscisses [qui m'intéresse], l'histoire se situe sur l'horizontale... je voudrais l'étirer dans le sens de la longueur ${ }^{48} \ldots$ ".

Vis-à-vis d'une conception hagiographique des avant-gardes japonaises comme ensemble de personnalités fortes qui ont inspiré, provoqué ou combattu les masses, il nous faut camper le contexte dans lequel elles furent actives : le topos qui a conditionné cette production. Cela conduit à minimiser l'effet d'opposition radicale ou de lutte entre des artistes inspirés et les masses. Enfin, bien qu'importé, l'underground japonais tel qu'il a pris racine à Shinjuku a rapidement développé des singularités qui méritent qu'on leur consacre une étude spécifique, et qui offrent de nouvelles clés de compréhension de la culture japonaise de l'après-guerre. En outre, l'underground a produit au Japon un style propre dans toutes les disciplines artistiques, immédiatement reconnaissable et désormais établi comme les autres styles artistiques. Il nourrit encore (sous une forme évidée et de manière formaliste, il faut bien le reconnaître) la culture visuelle japonaise, notamment le graphisme ${ }^{49}$. Cela n'avait pas échappé à l'agence de publicité japonaise Dentsū 電通 qui, dès mars 1968, avait mené sa propre enquête sur le potentiel commercial de l'angura ${ }^{50}$. 


\section{Kei Osawa}

Né en 1984 au Japon, ancien élève de l'École normale supérieure de Paris, Kei Osawa est chercheur en histoire de l'art et en esthétique au musée de l'Université de Tōkyō depuis 2008. Outre ses recherches sur les avant-gardes japonaises d'après-guerre, il travaille sur les Sound Studies et sur les débuts du film scientifique au Japon. Il a organisé de nombreuses expositions, à I'Intermediatheque de Tōkyō et ailleurs, sur les rapports entre arts et sciences.

\section{NOTES}

1. Chong Do-Ryun (dir.), Tokyo 1955-1970: A New AvantGarde, cat. exp. (New York, The Museum of Modern Art, 2013), New York, MoMA, 2012.

2. Ajia ni mezametara: āto ga kawaru, sekai ga kawaru 1960-1990 nendai アジアにめざめたらーアートが変わ る、世界が変わる 1960-1990年代 [Awakenings: Art in Society in Asia 1960s-1990s], cat. exp. (Tōkyō, Musée national d'art moderne de Tōkyō, 2018), Tōkyō, 2018.

3. Entre la France et le Japon, les « passeurs » ne manquent pas, tel Georges Mathieu séjournant au Japon, ou Kudō Tetsumi 工藤哲巳 vivant en France.

4. Tomii Reiko, "International Contemporaneity" in the 1960s: Discoursing on Art in Japan and Beyond", dans Japan Review, 2009, n 21, p. 123-147.

5. Nous traitons principalement de Tōkyō, mais il va de soi que l'essor des subcultures et contre-cultures ne s'est pas cantonné à la capitale. Voir Tomii Reiko, Radicalism in the Wilderness: International Contemporaneity and 1960s Art in Japan, Cambridge, MIT Press, 2016. Relevons notamment parmi les publications d'artistes un journal d'information spécialisé publié à Nagoya : Iwata Shin'ichi 岩田 信市 (dir.), Angura tsūshin アングラ通信 [Lettre d'information underground], nº 1 et 2, Nagoya, janvier-février 1969.

6. Shinohara Ushio 篠原有司男, Zen'ei no michi 前衛の 道 [La voie de l'avant-garde], Tōkyō, Bijutsu Shuppansha 美術出版社, 1969.

7. Sur la formation des « tribus » dans le Japon d'aprèsguerre, voir Nanba Kōji 難波功士, Zoku no keifugaku - Yūsu, sabukaruchāzu no sengoshi 族の系譜学一 ユース・サブカルチャーズの戦後史 [Généalogie des tribus : histoire de l'après-guerre selon les subcultures jeunes], Tōkyō, Seikyūsha 青弓社, 2007.

8. Gendai yōgo no kiso chishiki 現代用語の基礎 知識 [Connaissances fondamentales des termes contemporains], Tōkyō, Jiyū Kokumin-sha 自由国民社, 1969 (ma traduction).

9. Gendai yōgo no kiso chishiki, Tōkyō, Jiyū Kokuminsha 自由国民社, 1968, p. 1606.

10. C'est d'ailleurs le terme figurant dans le titre original du roman de Tanizaki Jun'ichirō, Journal d'un vieux fou (1961).

11. Akatsuka Yukio 赤塚行雄, Geba/An Goten ゲバ・ アン語典 [Lexique des termes gebaruto et angura], Tōkyō, Jiyū Kokumin-sha 自由国民社, 1969.
12. Sur les activités essentielles du Sōgetsu Art Center 草月アートセンター entre août 1958 et 1971, voir : Kagayake 60nendai - Sōgetsu āto sentā no zenkiroku 輝け60年代一草月アートセン夕の全記録 [Que les années 1960 étincellent : archives complètes du Sōgetsu Art Center], Tōkyō, Film Āto-sha フィルムアート社, 2002.

13. La traduction japonaise du manifeste du New American Cinema Group par Jonas Mekas (daté du 30 septembre 1960), déjà publiée l'année précédente, est également reprise en fin de livret.

14. Sheldon Renan, Andāguraundo eiga アンダーグラウンド 映画 [Le cinéma underground], Hatano Tetsurō 波多 野哲朗 (trad. jp.), Tōkyō, San-Ichi Shobō 三一書房, 30 juin 1969 [éd. orig. : An Introduction to the American Underground Film, New York, Dutton, 1967].

15. «Angura '68 » アングラ'68 [Underground 68], dans Bessatsu Kinema Junpō 別冊キネマ旬報, juin 1968.

16. «Angura '68 shokku-hen » アングラ'68 ショック篇 [Underground 68, volume choc], Bessatsu Kinema Junpō, août 1968.

17. À savoir Kanesaka Kenji, le réalisateur Adachi Masao 足立正生 (né en 1939), le dramaturge et écrivain Kara Jūrō 唐十郎 (né en 1940), le dramaturge, poète et cinéaste Terayama Shūji 寺山修司 (1935-1983), les actrices Niitaka Keiko 新高恵子 (née en 1934) et Yoshida Hideko 吉田日出子 (née en 1944), ainsi que les graphistes Yokoo Tadanori 横尾忠則 (né en 1936) et Uno Akira 宇野亜喜良 (né en 1934).

18. Tone Yasunao, «Nihon no andāguraundo āto »日本 のアンダーグラウンド・アート [L'art underground japonais], dans Bijutsu techō 美術手帖 n $^{\circ} 289$, novembre 1967, p. 98.

19. Hiraoka Masaaki 平岡正明, Angura kikansetsu Yami no hyōgensha retsuden アングラ機関説一闇の 表現者列伝 [Théorie de l'underground comme organisation : vies des créateurs de l'ombre], Tōkyō, Magajin Faibu マガジンファイブ, 2007, p. 195-200.

20. Tone, 1967, cité n. 18, p. 106 (ma traduction).

21. Uekusa Jin'ichi 植草甚—, «Andāguraundo komikkusu » アンダーグラウンド•コミックス [Underground Comics], dans Yuriika ユリイカ [Eurêka], vol. 1, nº 2, août 1969.

22. Michael Molasky, Sengo nihon no jazu bunka: eiga, bungaku, angura 戦後日本のジャズ文化一映画・文学・ アングラ [La culture jazz du Japon d'après-guerre : cinéma, littérature, underground], Tōkyō, Seidosha 青 土社, 2005.

23. Yoshioka Shinobu 吉岡忍, Fōku gerira towa nanimonoka フォーク・ゲリラとは何者か [Qui sont les folk guerilla ?], Tōkyō, Jiyū Kokuminsha 自由国民社, 1970.

24. Okumuro Minako 奥室美奈子 (dir.), Aa Shinjuku Spekutakuru toshite no toshi あ) 新宿一スペクタクル としての都市 [Ah Shinjuku - La ville comme spectacle], cat. exp. (Tōkyō, Musée du théâtre de l'Université Waseda 早稲田大学坪内博士記念演劇博物館), Tōkyō, 2016.

25. Nishidō Kōjin 西堂行人, Shōgen - Nihon no anguraEngeki kakumei no kishu tachi〈証言〉日本のアングラ 演劇革命の旗手たち [Témoignage : I'angura japonais Les porte-drapeaux de la révolution théâtrale], Tōkyō, Sakuhinsha 作品社, 2005. 
26. Ichikawa Miyabi 市川雅, « Angura engeki kikan zasshi »アングラ演劇機関雑誌 [Les bulletins du théâtre underground], dans SD Space Design, n 57, août 1969.

27. Takaguchi Yasuyuki 高口恭行 \& Manabe Tsunehiro 真鍋恒博, Daisan no kenchikuka - Angura ākitekuto 第三の建築家一アングラアーキテクト [Le troisième architecte : underground architect], Tōkyō, Shōkokusha 彰国社, 1972.

28. Taki Kōji 多木浩二, Henry Smith \& Isozaki Arata 磯崎 新, «Aimaisa no kūkan » あまいさの空間 [L'espace de l'ambiguïté], dans Asahi jānaru 朝日ジャーナル [Asahi Journal], vol. 17, n 14, 4 avril 1975, p. 27-31; Uchida Eiichi 内田栄一, Dezain wa kankyō o kaeru ka - Omoni Shinjuku o megutte デザインは環境を変えるか一おもに 新宿をめぐって [Le design va-t-il changer l'environnement ? Sur Shinjuku principalement], dans Dezain デザイン, no 134, juin 1970.

29. «Angura Tōkyō kōsō »アングラ東京構想 [Plan Tōkyō underground], dans Kenchiku bunka 建築文 化 [Culture architecturale], vol. 37, n ${ }^{\circ} 433$, novembre 1982 , p. $39-154$

30. Ibidem, p. 102.

31. "Les câbles aériens et les réseaux de canalisations, et puis le développement désordonné des passages souterrains - sur terre un nid d'araignée, sous terre une fourmilière. ", Ibidem, p. 103.

32. 大宅壮一Ōya Sōichi, «Angura wa atarashikunai » アングラは新しくない [L'angura n'est pas nouveau], dans Sandē Mainichi サンデー毎日 [Sunday Mainichi], 30 juin 1968 ; Fujieda Teruo 藤枝晃雄, "Angura no hisan to hisan »アングラの飛散と悲惨 [Éparpillement et misère de l'underground], dans SD Space Design, n 57, août 1969.

33. Sekine Hiroshi 関根弘, Waga Shinjuku ! - Hangyaku suru machi わが新宿! 一叛逆する町 [Notre Shinjuku! La ville qui se révolte], Zaikai Tenbōsha 財界展望社, 1969 ; Haga Zenjirō 芳賀善次郎, Shinjuku no konjaku 新宿の今昔 [Shinjuku aujourd'hui et autrefois], Tōkyō, Kinokuniya Shoten 紀伊國屋書店, 1970; Tanabe Moichi 田辺茂一, Waga machi Shinjuku わが町·新宿 [Ma ville, Shinjuku], Tōkyō, Sankei Shuppan サンケイ出版, 1976.

34. Guruppe 21 Seiki (dir.) グルッペ21世紀, Korega angura da! これがアングラだ! [Voici l'underground!], Tōkyō, Futaba-sha 双葉社, 1er juillet 1968.

35. Fukasaku Mitsusada 深作光貞, Shinjuku kōgengaku 新宿考現学 [Modernologie de Shinjuku], Tōkyō, Kadokawa shoten 角川書店, 15 septembre 1968.

36. Ce corpus immense est une mine d'informations sur les artistes concernés. Sur un ton plus prosaïque, voir : «Shinjuku fūten 30nin no shinjō zenchōsa » 新宿 フーテン30人の身上全調査 [Enquête sur 30 fūten de Shinjuku], dans Josei jishin 女性自身 [Les femmes ellesmêmes], vol. 12, nº 29, 2 août 1969.

37. Voir notamment les écrits de Kuzui Kinshirō 葛井 欣士郎, gérant de l'établissement: Kieta gekijō: āto shiatā shinjuku bunka 消えた劇場 アートシアター新宿 文化 [Le théâtre disparu : Art Theatre Shinjuku Bunka], Tōkyō, Sōryūsha 創隆社, 1984 ; Yuigon : āto shiatā shinjuku bunka 遺言 アートシアター新宿文化 [Testament : Art Theatre Shinjuku Bunka], Tōkyō, Kawade Shobō Shinsha 河出書房新社, 2008
38. Ōki Haruko 大木晴子 \& Suzuki Hitoshi 鈴木一 誌 (dir.), 1969 - Shinjuku nishiguchi chika hiroba 1969新宿西口地下広場 [1969 - La place souterraine à Shinjuku sortie ouest], Tōkyō, Shinjuku Shobō 新宿 書房, 2014.

39. "Shinjuku eki nishiguchi chika hiroba to sono shūhen » 新宿駅西口地下広場とその周辺 [La place souterraine de la sortie ouest de la gare de Shinjuku et ses environs], dans SD Space Design, n 37, décembre 1967.

40. Évoquée et parodiée par Tsutsui Yasutaka 筒井 康隆, dans «Shinjuku-sai » 新宿祭, Rippū Shobō 立風 書房, 1972.

41. Tōno Yoshiaki 東野芳明, "Shinjuku nishiguchi ‘hiroba' no seitaigaku » 新宿西口“広場”の生態学 [Écologie de la " place » de Shinjuku ouest], dans Chūō Kōron 中央公論, octobre 1969, Tōkyō, Chūō Kōronsha 中 央公論社.

42. Fukuda Seiken 福田晴虔, «Kenchiku dezain » 建築 デザイン [L'architecture et le design], « Nihon bankoku hakurankai no seika »日本万国博覧会の成果 [Les fruits de l'Exposition universelle au Japon], dans Kenchiku zasshi 建築雑誌, vol. 85, n 1030, novembre 1970.

43. "Gendai no hiroba - Shinjuku / Kinō no fūten wa doko e » 現代の広場一新宿/昨日のフーテンはどこへ [La place contemporaine - Shinjuku / Où sont les fūten d'hier ?], dans Sandē Mainichi サンデー毎日 [Sunday Mainichi], 15 juin 1969.

44. Outre Moriyama Daidō, relevons notamment Tōmatsu Shōmei 東松照明, Oo! Shinjuku おお! 新宿, Tōkyō, Shaken 写研, 1969; Watanabe Hitomi 渡辺眸, 1968 Shinjuku 1968新宿, Tōkyō, Machikarasha 街 から舎, 2014 ; Hanaga Mitsutoshi 羽永光利, Mitsutoshi Hanaga 1000, Tōkyō, 1000 Bunko, 2017.

45. Sur l'inscription de ces deux films dans Shinjuku, voir Taro Nettleton, "Shinjuku as Site: Funeral Parade of Roses and Diary of a Shinjuku Thief ", dans Screen, vol. 55, n 1, p. 5-28, 2014.

46. Kanesaka Kenji (dir.), Andāguraundo generēshon アンダーグラウンド・ジェネレーション [Underground Generation], Tōkyō, Nōberu Shobō ノーベル書房, 1968.

47. Voir à ce sujet Miyazawa Akio 宮沢章夫, Nippon sengo sabukaruchā-shi ニッポン戦後サブカルチャー史 [Histoire de la subculture du Japon d'après-guerre], Tōkyō, NHK Shuppan NHK出版, 2014, p. 33-51.

48. Tone, 1967, cité n. 18, p. 106.

49. Collectif, Angura - Nihon no posutā no avangyarudo 1960-1980 アングラ 日本のポスターのアヴアンギャルド 1960-1980 [Underground: L'avant-garde des affiches au Japon 1960-1980], Tōkyō, Bibliothèque de documentation artistique de l'Université d'art de Musashino 武蔵野美術大学美術資料図書館, 1999.

50. Mishima Akira 三島彰, Abangyarudo ninen no kankaku kōzō アバンギャルド二年の感覚構造 [La structure sensorielle de l'avant-garde à sa deuxième année], dans Māketingu to kōkoku マーケティングと広告 [Marketing et publicité], mars 1968, Tōkyō, Dentsū 電通. 